\title{
Potential of bone marrow mesenchymal stem cells in rejuvenation of the aged skin of rats
}

\author{
ZHEN LIU $^{1 *}$, GUO-DONG HU $^{1 *}$, XIAO-BO LUO ${ }^{2 *}$, BIN YIN $^{1}$, BIN SHU ${ }^{1}$, JING-ZHI GUAN ${ }^{3}$ and CHI-YU JIA ${ }^{1}$ \\ Departments of ${ }^{1}$ Burns and Plastic Surgery, ${ }^{2}$ Orthopedics and ${ }^{3}$ Oncology, \\ The 309th Hospital of PLA, Beijing 100091, P.R. China
}

Received August 8, 2016; Accepted September 23, 2016

DOI: $10.3892 /$ br. 2017.842

\begin{abstract}
The aim of the present study was to evaluate the anti-aging effects of bone marrow-mesenchymal stem cells (BM-MSCs) in a D-galactose-induced skin aging rat model. Male Sprague Dawley rats were randomly divided into four groups (n=10/group) as follows: Normal control group; skin aging model group; MSC-treated group by subcutaneous multi-point injection. The skin aging model was established by a daily subcutaneous injection of $15 \%$ D-galactose $(1,000 \mathrm{mg} / \mathrm{kg})$ for 8 weeks. Rats in the MSC-treated groups were administered $3 \times 10^{6} / \mathrm{ml}$ BM-MSCs/green fluorescent protein (GFP) for 4 weeks, administered once per week. Oxidative/antioxidative parameters were evaluated, and morphological and ultrastructure analyses were performed. Rats in the model group exhibited the typical changes of aging skin. Compared with the control group, rats in the model group had significantly increased malondialdehyde (MDA) content $(\mathrm{P}<0.01)$, and decreased serum superoxide dismutase (SOD) and glutathione peroxidase (GSH-Px) activities $(\mathrm{P}<0.05)$. MSC treatment markedly ameliorated aging-induced oxidative stress in the skin. Histologically, rats in the model group exhibited loosely arranged epidermal cell layers and disorganized collagen fibers. BM-MSC treatment significantly improved the histological abnormalities, which was similar to those in the control group. In addition, 7 days after the final cell transplantation, GFP-positive cells were observed by fluorescence microscopy to be distributed in the dermis. Injection of BM-MSCs significantly improved the
\end{abstract}

Correspondence to: Professor Chi-Yu Jia, Department of Burns and Plastic Surgery, The 309th Hospital of PLA, 17 Heishanhu Street, Beijing 100091, P.R. China

E-mail: lzddm329@126.com

Professor Jing-Zhi Guan, Department of Oncology, The 309th Hospital of PLA, 17 Heishanhu Street, Beijing 100091, P.R. China E-mail: 1602877837@qq.com

${ }^{*}$ Contributed equally

Key words: mesenchymal stem cells, aging, skin, oxidative stress
D-galactose-induced histological abnormalities of the skin, by promoting an antioxidant response and ameliorating oxidative stress in aged skin. Thus, BM-MSCs may be beneficial in the rejuvenation of aged skin.

\section{Introduction}

Skin maintains various homeostatic processes of the body and acts as the first defense barrier against infection, radiation and toxin exposure. However, skin is easily affected by the natural aging process (i.e., intrinsic aging), as well as environmental conditions (i.e., extrinsic aging), undergoing aging-associated phenotypic and functional alterations in the cellular and extracellular components $(1,2)$. Skin aging is a biological, inevitable process accompanied by a decreased regenerative capacity, loss of dermal elasticity, impaired function of the skin, and increased risk of cancer (3). Histologically, aging of the skin results in altered epidermal architecture and morphology, reduced dermal mast cells and fibroblasts, decreased collagen production, epidermal thinning and diminished dermis vasculature (4,5). Mechanisms for aging skin include exacerbated formation of free radicals (reactive oxygen species; ROS), accumulation of mitochondrial DNA mutations, progressive telomere shortening, ultraviolet (UV) radiation, inflammation and hormonal changes, as well as other factors that, taken together or alone, may accelerate skin aging (6-8). However, currently there are a variety of anti-aging agents $(7,8)$, which are routinely used, although their therapeutic efficiency is less than satisfactory. Therefore, novel therapeutic strategies for the rejuvenation of aged skin are urgently required.

Mesenchymal stem cells (MSCs) are rare multipotent stem cells of mesenchymal origin. Through identification with specific surface markers, MSCs are isolated from the majority of human tissue types, including bone marrow, adipose tissues, umbilical cord blood and the dermis (9). MSCs derived from bone marrow (BM-MSCs) possess remarkable self-renewing properties and exhibit a multipotent differentiation profile under appropriate differentiation conditions in vivo and in vitro $(10,11)$. MSCs facilitate tissue repair via cell replacement from differentiated cells and/or remodeling the microenvironment by releasing chemokines and growth factors. MSCs, therefore, facilitate a variety of basic and clinical studies for the treatment of a plethora of congenital and acquired diseases (12). MSCs have been reported to 
accelerate the healing of cutaneous wounds by systemic and/or local administration (13-15). Animal experiments have confirmed the anti-apoptotic and anti-oxidative capacities of MSCs (16), therefore, these cells may be considered as an alternative therapeutic strategy for skin rejuvenation. Conditioned serum-free medium from umbilical cord-derived MSCs may protect against photo-aging induced by UV radiation in mouse skin (17). Furthermore, adipose-derived MSCs have been reported to improve UV radiation-induced wrinkles and protect photo-aged dermal fibroblasts from oxidative stress $(18,19)$.

However, the underlying mechanisms by which BM-MSCs exert their anti-aging effects have not been extensively investigated. The aim of the current study was to evaluate the anti-aging effects of BM-MSCs (using a skin aging model induced by intradermal injection of D-galactose), as well as the underlying mechanisms.

\section{Materials and methods}

Experimental animals. Thirty male Sprague-Dawley (SD) rats (age, 4-6 weeks), weighing $200 \pm 20 \mathrm{~g}$, were purchased from the Experimental Animal Center, the General Hospital of the Chinese People Liberation Army (PLA; Beijing, China). They were maintained separately in a constant environment (room temperature, $20-22^{\circ} \mathrm{C}$; room humidity, $40-60 \%$, with free access to food and water) under a 12-h light/dark cycle. All procedures were performed with the approval of the Committee on the Use of Live Animals in Teaching and Research of the 309th Hospital of PLA.

Preparation and culture of rat BM-MSCs. BM-MSCs with green fluorescent protein (GFP) from SD rats were purchased from Cyagen Biosciences (Santa Clara, CA, USA). The cells were centrifuged at $500 \mathrm{xg}\left(20-22^{\circ} \mathrm{C}\right)$ for $5 \mathrm{~min}$ and suspended in $\alpha$-Minimum Essential Medium (Thermo Fisher Scientific, Grand Island, NY, USA) supplemented with Gibco 10\% fetal bovine serum (Thermo Fisher Scientific) and then incubated at $37^{\circ} \mathrm{C}$ with $5 \% \mathrm{CO}_{2}$. The cells were passaged at a ratio of $1: 2$ with $0.25 \%$ trypsin (Thermo Fisher Scientific), reseeded into new flasks and cultured until reaching $80 \%$ confluence. The fourth generation of BM-MSCs/GFP was collected, suspended in phosphate-buffered saline (PBS) and used to inject the rats.

Establishment of the rat model of skin aging. SD rats were randomly divided into three groups ( $\mathrm{n}=10 /$ group) as follows: Normal control group; aging model group; MSC-treated group (subcutaneous injection). The rats in the control group received a daily subcutaneous injection of sterile saline $(1 \mathrm{ml})$, and the other three groups were administered a daily subcutaneous injection of $15 \%$ D-galactose $(1,000 \mathrm{mg} / \mathrm{kg}$ in $1 \mathrm{ml}$ sterile saline) for 8 weeks (20).

In vivo transplantation of BM-MSCs. Following successful preparation of the rat model of skin aging, rats in the MSC-treated groups were given $3 \times 10^{6} / \mathrm{ml} \mathrm{BM-MSCs/GFP} \mathrm{in}$ $1 \mathrm{ml}$ PBS by subcutaneous multi-point injection at the midline of the dorsum for 4 weeks, which was administered once per week. Rats in the control and model groups were given the same quantity of PBS according to the same routine at the same time points. All animals were allowed free access to water and food, and were observed daily to assess the general condition of the rats.

Fluorescence analysis for labeling and trafficking of BM-MSCs in skin. The mice were sacrificed by cervical dislocation after the experiments, and the skin tissue was immediately collected and prepared for cryosectioning. Frozen sections (thickness, $30 \mu \mathrm{m})$ were sliced from the skin tissues of the dorsum and the GFP-positive cells were observed under a fluorescence microscope (IX-51; Olympus Corporation, Tokyo, Japan).

Morphological and ultrastructure analysis. Skin tissue samples from rats in each group were obtained and fixed in 4\% paraformaldehyde for $12 \mathrm{~h}$, then embedded in paraffin, and sliced into $5-\mu \mathrm{m}$ sections. The sections were mounted on glass slides, dewaxed, rehydrated with distilled water, stained with hematoxylin and eosin, and observed under a light microscope. Van Gieson stain was used for collagen fiber staining.

For ultrastructural examination by transmission electron microscopy (TEM; FEI Tecnai ${ }^{\mathrm{TM}}$, Hillsboro, OR, USA), the skin tissue samples from rats were fixed in $2.5 \%$ pentanediol-2\% paraformaldehyde in $0.1 \mathrm{~mol} / 1$ sodium phosphate for $12 \mathrm{~h}$ at $4^{\circ} \mathrm{C}$. After three washes with PBS, the samples were then secondarily fixed in $1 \%$ osmium tetroxide (osmic acid) for $1.5 \mathrm{~h}$. The samples were thoroughly dehydrated in solutions of ethanol at increasing concentrations of 50, 70, 80, 90 and $100 \%$ for 10 min each, followed by immersion in 50\% acetone for $1 \mathrm{~h}$ and in $33 \%$ acetone for $3 \mathrm{~h}$. The samples were transferred into fresh epoxy resin and polymerized at $60^{\circ} \mathrm{C}$ for $48 \mathrm{~h}$. Finally, the fixed tissues were thin-sectioned to $60-80 \mathrm{~nm}$ using an ultramicrotome and subsequently subjected to TEM for ultrastructural study.

Measurements of oxidant/antioxidant parameters. Skin tissue samples were homogenized in normal saline, and sonicated twice to produce homogenates, followed by centrifugation at $1,000 \times \mathrm{g}\left(20-22^{\circ} \mathrm{C}\right)$ for $10 \mathrm{~min}$ and $4,000 \times \mathrm{g}\left(4^{\circ} \mathrm{C}\right)$ for $15 \mathrm{~min}$. The supernatant was collected and total proteins were quantified using the bicinchonic acid (BCA) method. The malondialdehyde (MDA) content, and superoxide dismutase (SOD) and glutathione peroxidase (GSH-Px) activities were determined using a total-SOD (T-SOD) assay kit (cat. no. A001-1), a MDA assay kit (cat. no. A003-1) and a GSH-PX assay kit (cat. no. A0005) according to the manufacturer's instructions (Jiancheng Biotech Co., Ltd., Nanjing, China). Briefly, the SOD activity was measured using the xanthine oxidase method at a wavelength of $550 \mathrm{~nm}$, the MDA content was determined using the thiobarbituric acid colorimetric method at a wavelength of $532 \mathrm{~nm}$ and the GSH-Px activity was detected using the chemical colorimetric method at a wavelength of $412 \mathrm{~nm}$. These oxidant/antioxidant indicators (SOD, MDA and GSH-Px activity) were measured using a 752 UV spectrophotometer (Hitachi, Ltd., Tokyo, Japan).

Statistical analysis. Statistical analysis was performed using SPSS software (version 12.0; SPSS, Inc, Chicago, IL, USA) and the quantitative data are presented as the mean \pm standard deviation. Comparisons between two groups were performed 
Table I. Comparison of oxidant/antioxidant parameters among groups ( $\mathrm{n}=10 /$ group).

\begin{tabular}{|c|c|c|c|}
\hline \multirow[b]{2}{*}{ Parameter } & \multicolumn{3}{|c|}{ Group (means \pm standard deviation) } \\
\hline & Control & Model & Mesenchymal stem cell-treated \\
\hline Malondialdehyde content (nmol/ml) & $5.7 \pm 0.6$ & $13.2 \pm 0.9^{\mathrm{a}}$ & $8.6 \pm 0.5^{\mathrm{b}}$ \\
\hline Superoxide dismutase activity $(\mathrm{nU} / \mathrm{ml})$ & $148.1 \pm 10.1$ & $95.0 \pm 7.5^{\mathrm{c}}$ & $132.8 \pm 8.3^{\mathrm{d}}$ \\
\hline Glutathione-Peroxidase activity (U/ml) & $236.1 \pm 18.3$ & $169.0 \pm 11.5^{\mathrm{c}}$ & $201.9 \pm 15.1^{\mathrm{d}}$ \\
\hline
\end{tabular}

${ }^{\text {a }} \mathrm{P}<0.01$ vs. control, ${ }^{\mathrm{b}} \mathrm{P}<0.05$ vs. model, ${ }^{\mathrm{c}} \mathrm{P}<0.05$ vs. control, ${ }^{\mathrm{d}} \mathrm{P}<0.05$ vs. model.

by unpaired Student's t-test and $\mathrm{P}<0.05$ was considered to indicate a statistically significant difference.

\section{Results}

General condition of the animals. The rats in the model group showed lower mood, reduced appetite and water consumption, reduced activity, dull fur and decreased skin elasticity in comparison with the rats in the control group. However, the MSC-treated groups showed improvement of these symptoms.

Comparison of oxidant/antioxidant parameters among groups. Compared with the control group, rats in the model group had a significantly increased MDA content $(\mathrm{P}<0.01)$, and significantly decreased serum GSH-Px and SOD activities (Table I; P<0.05). Treatment with 3x106 BM-MSCs by subcutaneous injection led to a significant increase in serum GSH-Px and SOD activities $(\mathrm{P}<0.05)$ and decrease in MDA content when compared with the model group $(\mathrm{P}<0.01)$, indicating that these treatments markedly ameliorated aging-induced oxidative stress in skin.

Trafficking of BM-MSCs in skin. Seven days after the final cell transplantation, the GFP-positive cells were distributed in the dermis, which was observed by fluorescence microscopy (Fig. 1).

Histological and electron micrographic findings. Histological sections of skin tissue samples were stained with hematoxylin and eosin or Van Gieson stain, and examined microscopically (Fig. 2). Skin sections from the control group showed normal epidermal morphology with regular arrangement of epidermal cell layers and normal integral structures of the dermis. Collagen fibers were neatly and densely arranged, and interwoven into a network. Rats in the model group exhibited loosely arranged epidermal cell layers and disorganized collagen fibers. Following BM-MSC treatment for 4 weeks, the histological abnormalities significantly improved, which was evidenced by almost normal epidermal morphology and densely arranged collagen fibers, which were similar to those observed in the control group.

To assess the protective effects of BM-MSCs on aging-induced skin lesions, ultrastructural analysis by TEM was performed on the skin tissue samples (Fig. 3). Rats in the control group showed abundant, well-developed rough endoplasmic reticulum and fibroblasts, surrounded by normal
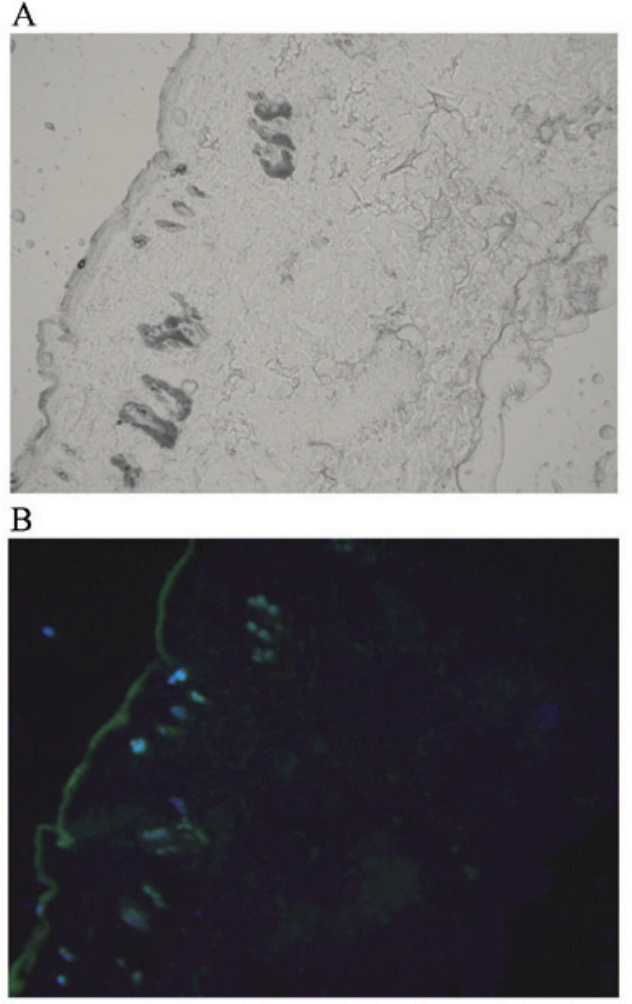

Figure 1. Distribution of green fluorescent protein-positive cells in the dermis. The sections of skin were examined under (A) light and (B) fluorescence microscopes 7 days after the final bone marrow mesenchymal stem cell transplantation (magnification, $\mathrm{x} 100$ ).

collagen fibers of dense connective tissue, which were arranged parallel to each other in neat rows within the dermal layer. In the model group, the number of rough endoplasmic reticulum was significantly reduced. The bundles of collagen fibers lost their regular arrangement and presented as a loosely connected network. In addition, broken or dissolved fibers were observed. Notably, subsequent to 4-week BM-MSC treatment, the rats demonstrated marked improvements in the ultrastructural abnormalities. Rough endoplasmic reticulum with normal appearance were clearly visible and the close arrangement of dermal collagen fibers was similar to those in the control group.

\section{Discussion}

The present study provides evidence for the anti-aging effects of BM-MSCs against D-galactose-induced skin aging, as 
H\&E staining

A

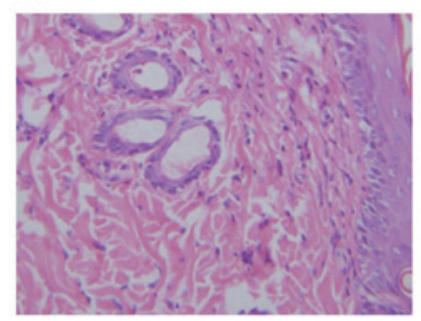

B

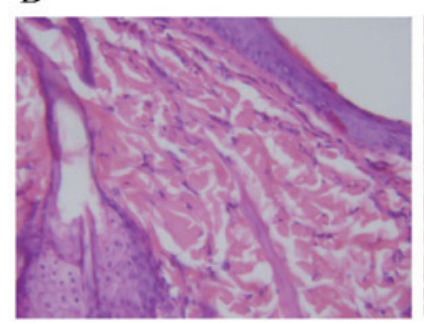

C

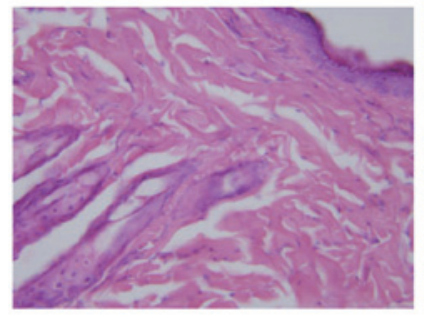

Van Gieson staining
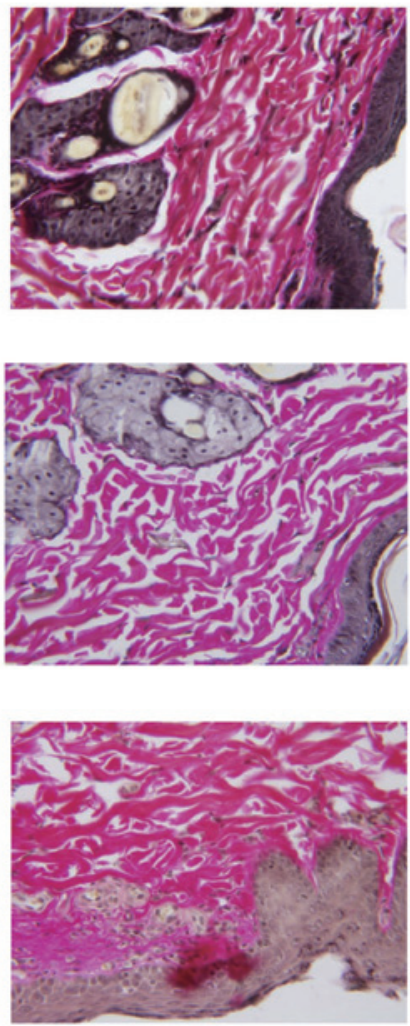

Figure 2. Histological findings of skin tissue samples examined using hematoxylin and eosin (H\&E) staining, and under light microscopy. (A) In the control group, following Van Gieson staining, the skin sections demonstrated normal epidermal morphology with regular arrangement of epidermal cell layers and normal integral structures of the dermis. The collagen fibers were neatly and densely arranged. (B) In the model group, the skin sections showed loosely arranged epidermal cell layers and disorganized collagen fibers. (C) In the bone marrow mesenchymal stem cell-treated group, the histological abnormalities were significantly improved, as evidenced by neatly and densely arranged collagen fibers, which were similar to those observed in the control group. Magnification, $x 100$.

evidenced by substantial improvement in histological and ultrastructural abnormalities, decreased expression of the oxidant-associated marker, MDA and increases in the antioxidant markers, SOD and GSH-Px. In addition, the BM-MSCs were retained 7 days after being injected into dermal tissues.

The dermal structure is primarily composed of fibroblasts and extracellular matrix, which is rich in collagen and elastic fibers. The collagen-rich extracellular matrix is synthesized, organized and maintained by dermal fibroblasts. With age, fibroblast cells gradually decrease in number, and exhibit diminished capacity to synthesize collagen and elastic proteins, resulting in laxity and decreased skin elasticity. Histological and ultrastructural studies have revealed major alterations in the dermal extracellular matrix in aged skin, with reduced fibroblasts and decreased collagen production (5). Chronic injection with D-galactose at a wide range of dosages has been used to establish a skin aging model in rodents that resembles intrinsic aging, characterized by typical changes of aging skin in behavior, phenotype, histological appearance, as well as expression of senescence markers (e.g., SOD and MDA) (20-22). In the current study, rats in the model

A
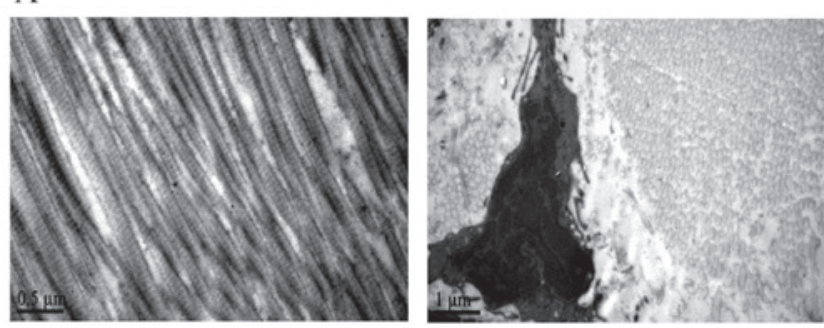

$\mathrm{B}$
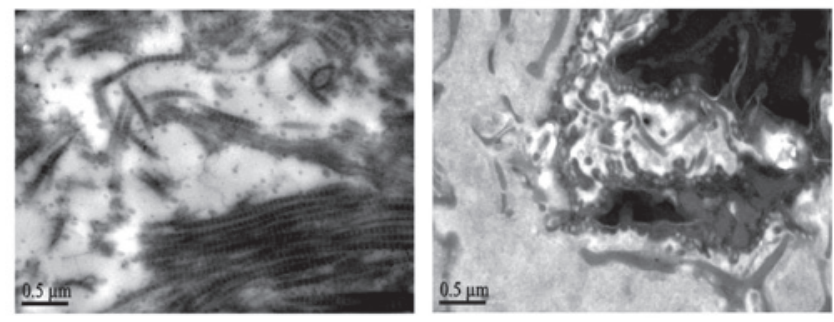

$\mathrm{C}$
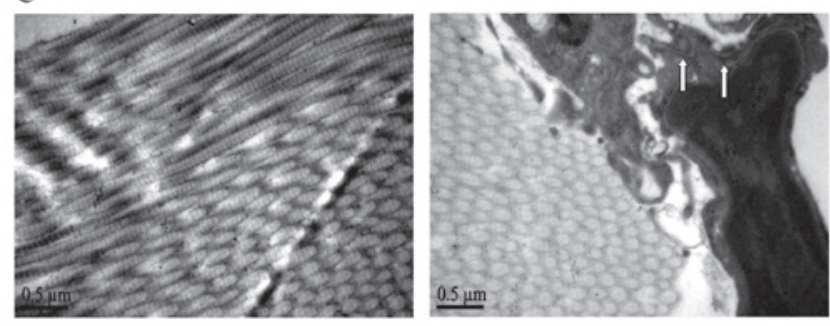

Figure 3. Ultrastructural analysis of skin tissue samples using transmission electron microscopy. (A) Samples from the control group demonstrate abundant, well-developed rough ER and fibroblasts, surrounded by neatly arranged collagen fibers within the dermal layer. (B) Samples from the model group demonstrated a significantly reduced number of rough ER. The bundles of collagen fibers (including those that were broken or dissolved) were no longer neat and regularly arranged, and presented a loosely knit network. (C) In the samples from the bone marrow MSC-treated group, the MSCs restored the normal ER morphology and improved the ultrastructural abnormalities. ER, endoplasmic reticulum; MSC, mesenchymal stem cell.

group were administered a daily subcutaneous injection of D-galactose $(1,000 \mathrm{mg} / \mathrm{kg})$ for 8 weeks. These rats showed poor mood, reduced appetite and water consumption, reduced activity, dull fur and decreased skin elasticity. In addition to typical symptoms of skin aging, this model appeared to exhibit an increase in MDA content and decrease in SOD and GSH-Px activity, and pathological characteristics of aged skin (including loosely arranged epidermal cell layers and disorganized collagen fibers) suggesting the successful establishment of a skin aging model in SD rats in this study.

The beneficial effect of MSCs on disease has been investigated in a variety of animal models and clinical trials. In particular, BM-MSCs contribute to cutaneous wound healing by promoting epidermal regeneration, as well as accelerating endothelial cell formation $(23,24)$. BM-MSCs were originally described as plastic-adherent fibroblast-like cells that are capable of differentiating into a variety of cell types, including epidermal cells, endothelial cells and fibroblasts in the presence of appropriate factors in vitro and in vivo (15). Furthermore, MSCs enhance the functions of keratinocytes and dermal fibroblasts in a paracrine fashion (25). Thus, the potential of BM-MSCs in the rejuvenation of aged skin 
were evaluated. In the present study, BM-MSC treatment for 4 weeks restored D-galactose-induced histological abnormalities to levels similar to those of the controls. The present study hypothesized that through releasing cytokines and growth factors, such as vascular endothelial growth factor, platelet-derived growth factor, transforming growth factor- $\beta$, BM-MSCs promote the proliferation of fibroblasts, which stimulate production of collagen and elastic fibers (25). As a consequence, transplantation of BM-MSCs results in the activation of fibroblasts, enhancement of extracellular matrix, and thereby increases skin elasticity and reduces the appearance of wrinkles. Indeed, experimental studies of cutaneous wounds treated with MSCs revealed a relatively low level of direct engraftment of transplanted cells into the epidermis, with fewer cells retained over time (15). The low engraftment rate of transplanted cells and short-term retention rates are currently considered as barriers that potentially diminish the benefits of cell therapy (26). Thus, repeated transplants are proposed to enable the maximum therapeutic effect of MSCs. In the current study, rats in the MSC-treated groups were administered $3 \times 10^{6} / \mathrm{ml} \mathrm{BM-MSCs}$ for 4 weeks, once per week. Seven days after the final cell transplantation, GFP-positive cells were observed to be distributed in the dermis under a fluorescence microscope. Consistently, MSCs have been reported to be detectable at 28 days after being injected into dermal tissues despite a low retention of transplanted cells (21). These data further indirectly confirm the anti-aging effects of MSCs in skin.

Oxidative stress is currently considered as a fundamental causative factor of aging, leading to intracellular lipid peroxidation, abnormal protein oxidation reactions and the accumulation of oxidative cellular damage (6). Aging appears to be a consequence of the imbalance between overwhelming ROS production beyond the scavenging ability, and substantial reduction in antioxidant defense during the aging process (27). Thus, attempts have been made to reduce excessive ROS in aged skin with the aim of improving, or even rejuvenating, aged skin (28). ROS degrade polyunsaturated lipids to form MDA, causing peroxidative tissue damage. SOD, an important antioxidant enzyme, functions to catalyze the dismutation of superoxide radicals to hydrogen peroxide and oxygen, defending the skin against ROS-induced oxidative deterioration of DNA, proteins and lipids by scavenging remaining ROS in cells (27). GSH-Px is a glutathione peroxidase that functions as an anti-oxidant by catalyzing the reduction of hydrogen peroxide to water and preventing lipid peroxidation (29). Zhang et al (21) demonstrated that D-galactose induced oxidative stress in mouse skin, as shown by the expression levels of increased MDA and decreased SOD. Consistent with these findings, the current study demonstrated that skin D-galactose-induced aging produced large quantities of lipid peroxide MDA accompanied by a decrease in the antioxidant enzymes, SOD and GSH-Px. Notably, MSCs have been reported to exhibit antioxidative activity under various conditions, by increasing the SOD and glutathione levels, and modulating the activation of antioxidant-associated proteins (30). Furthermore, MSCs exhibit potent antioxidant effects that provide protection for dermal fibroblasts and keratinocytes against oxidative stress, and consequently accelerate skin wound healing (31-33).
In the present study, BM-MSCs were shown to cause a greater increase in serum GSH-Px and SOD activities, and a significant decrease in MDA content when compared with the model group. This indicates that BM-MSCs promote an antioxidant response and ameliorate aging-induced oxidative stress in the skin.

In conclusion, injection of BM-MSCs significantly improve D-galactose-induced histological and ultrastructural abnormalities of the skin, by promoting an antioxidant response and by ameliorating aging-induced oxidative stress in aged skin. Thus, BM-MSCs may be beneficial in the rejuvenation of aged skin. However, further investigation is required to determine the exact molecular mechanisms underlying the antioxidant effects of MSCs.

\section{Acknowledgements}

The present study was supported by the National Natural Science Foundation Project (grant nos. 81372051 and 81670009), the Medical and Senile Disease Foundation Project of General Staff Headquarters (grant no. ZCWS14B06), the Beijing Science and Technology 'Capital Special' Program (grant no.Z151100004015199), and the Military Medical Science and Technology Youth Training Program (grant no. 15QNP049).

\section{References}

1. Quan T and Fisher GJ: Role of age-associated alterations of the dermal extracellular matrix microenvironment in human skin aging: A mini-review. Gerontology 61: 427-434, 2015.

2. Gunin AG, Kornilova NK, Vasilieva OV and Petrov VV: Age-related changes in proliferation, the numbers of mast cells, eosinophils, and cd45-positive cells in human dermis. J Gerontol A Biol Sci Med Sci 66: 385-392, 2011.

3. McCullough JL and Kelly KM: Prevention and treatment of skin aging. Ann N Y Acad Sci 1067: 323-331, 2006.

4. Hwang KA, Yi BR and Choi KC: Molecular mechanisms and in vivo mouse models of skin aging associated with dermal matrix alterations. Lab Anim Res 27: 1-8, 2011.

5. Varani J, Dame MK, Rittie L, Fligiel SE, Kang S, Fisher GJ and Voorhees JJ: Decreased collagen production in chronologically aged skin: Roles of age-dependent alteration in fibroblast function and defective mechanical stimulation. Am J Pathol 168: 1861-1868, 2006.

6. Cui H, Kong Y and Zhang H: Oxidative stress, mitochondrial dysfunction, and aging. J Signal Transduct 2012: 646354, 2012.

7. Panich U, Sittithumcharee G, Rathviboon N and Jirawatnotai S: Ultraviolet radiation-induced skin aging: The role of DNA damage and oxidative stress in epidermal stem cell damage mediated skin aging. Stem Cells Int 2016: 7370642, 2016.

8. Bhatia-Dey N, Kanherkar RR, Stair SE, Makarev EO and Csoka AB: Cellular senescence as the causal nexus of aging. Front Genet 7: 13, 2016.

9. Lv FJ, Tuan RS, Cheung KM and Leung VY: Concise review: The surface markers and identity of human mesenchymal stem cells. Stem Cells 32: 1408-1419, 2014.

10. Chamberlain G, Fox J, Ashton B and Middleton J: Concise review: Mesenchymal stem cells: Their phenotype, differentiation capacity, immunological features, and potential for homing. Stem Cells 25: 2739-2749, 2007.

11. Pittenger MF, Mackay AM, Beck SC, Jaiswal RK, Douglas R, Mosca JD, Moorman MA, Simonetti DW, Craig S and Marshak DR: Multilineage potential of adult human mesenchymal stem cells. Science 284: 143-147, 1999.

12. Wang J, Liao L and Tan J: Mesenchymal-stem-cell-based experimental and clinical trials: Current status and open questions. Expert Opin Biol Ther 11: 893-909, 2011.

13. Isakson M, de Blacam C, Whelan D, McArdle A and Clover AJ: Mesenchymal Stem Cells and Cutaneous Wound Healing: Current Evidence and Future Potential. Stem Cells Int 2015: 831095, 2015. 
14. Hocking AM and Gibran NS: Mesenchymal stem cells: Paracrine signaling and differentiation during cutaneous wound repair. Exp Cell Res 316: 2213-2219, 2010.

15. Chen JS, Wong VW and Gurtner GC: Therapeutic potential of bone marrow-derived mesenchymal stem cells for cutaneous wound healing. Front Immunol 3: 192, 2012.

16. Liu H, McTaggart SJ, Johnson DW and Gobe GC: Original article anti-oxidant pathways are stimulated by mesenchymal stromal cells in renal repair after ischemic injury. Cytotherapy 14: $162-172,2012$.

17. Liu Q, Luo Z, He S, Peng X, Xiong S, Wang Y, Zhong X, Zhou X, Eisenberg CA and Gao BZ: Conditioned serum-free medium from umbilical cord mesenchymal stem cells has anti-photoaging properties. Biotechnol Lett 35: 1707-1714, 2013.

18. Kim WS, Park BS, Park SH, Kim HK and Sung JH: Antiwrinkle effect of adipose-derived stem cell: Activation of dermal fibroblast by secretory factors. J Dermatol Sci 53: 96-102, 2009.

19. Kim WS, Park BS and Sung JH: Protective role of adipose-derived stem cells and their soluble factors in photoaging. Arch Dermatol Res 301: 329-336, 2009.

20. Zhen YZ, Lin YJ, Li KJ, Zhang GL, Zhao YF, Wang MM, Wei JB, Wei $\mathrm{J}$ and $\mathrm{Hu} \mathrm{G}$ : Effects of rhein lysinate on D-galactose-induced aging mice. Exp Ther Med 11: 303-308, 2016.

21. Zhang S, Dong Z, Peng Z and Lu F: Anti-aging effect of adipose-derived stem cells in a mouse model of skin aging induced by D-galactose. PLoS One 9: e97573, 2014.

22. Wei H, Li L, Song Q, Ai H, Chu J and Li W: Behavioural study of the $\mathrm{D}$-galactose induced aging model in $\mathrm{C} 57 \mathrm{BL} / 6 \mathrm{~J}$ mice. Behav Brain Res 157: 245-251, 2005.

23. Wu Y, Zhao RC and Tredget EE: Concise review: Bone marrow-derived stem/progenitor cells in cutaneous repair and regeneration. Stem Cells 28: 905-915, 2010.

24. Aoki S, Toda S, Ando T and Sugihara H: Bone marrow stromal cells, preadipocytes, and dermal fibroblasts promote epidermal regeneration in their distinctive fashions. Mol Biol Cell 15: 4647-4657, 2004.
25. Lee SH, Jin SY, Song JS, Seo KK and Cho KH: Paracrine effects of adipose-derived stem cells on keratinocytes and dermal fibroblasts. Ann Dermatol 24: 136-143, 2012.

26. Cassino TR, Drowley L, Okada M, Beckman SA, Keller B, Tobita K, Leduc PR and Huard J: Mechanical loading of stem cells for improvement of transplantation outcome in a model of acute myocardial infarction: The role of loading history. Tissue Eng Part A 18: 1101-1108, 2012.

27. Treiber N, Maity P, Singh K, Ferchiu F, Wlaschek M and Scharffetter-Kochanek K: The role of manganese superoxide dismutase in skin aging. Dermatoendocrinol 4: 232-235, 2012.

28. Chen H, Long Y and Guo L: Antiaging Effect of Inula britannica on Aging Mouse Model Induced by D-Galactose. Evid Based Complement Alternat Med 2016: 6049083, 2016.

29. Sandhu SK and Kaur G: Alterations in oxidative stress scavenger system in aging rat brain and lymphocytes. Biogerontology 3 : 161-173, 2002.

30. Kim WS, Park BS and Sung JH: The wound-healing and antioxidant effects of adipose-derived stem cells. Expert Opin Biol Ther 9: 879-887, 2009.

31. Kim Y, Jo SH, Kim WH and Kweon OK: Antioxidant and anti-inflammatory effects of intravenously injected adipose derived mesenchymal stem cells in dogs with acute spinal cord injury. Stem Cell Res Ther 6: 229, 2015

32. Ayatollahi M, Hesami Z, Jamshidzadeh A and Gramizadeh B Antioxidant Effects of Bone Marrow Mesenchymal Stem Cell against Carbon Tetrachloride-Induced Oxidative Damage in Rat Livers. Int J Organ Transplant Med 5: 166-173, 2014.

33. Zhuo W, Liao L, Xu T, Wu W, Yang S and Tan J: Mesenchymal stem cells ameliorate ischemia-reperfusion-induced renal dysfunction by improving the antioxidant/oxidant balance in the ischemic kidney. Urol Int 86: 191-196, 2011. 